\title{
An exploratory study to understand and examine the nature and type of relationship between self-esteem, life satisfaction and adjustment among male and female migrant students
}

\author{
Renu Seshadri ${ }^{1}$, Radhika Srinivasan ${ }^{2}$, Veena Kumar ${ }^{3}$ \\ ${ }^{1}$ Department of Psychology, KC College, Churchgate, Mumbai. \\ ${ }^{2}$ Department of English, KC College, Churchgate, Mumbai. \\ ${ }^{3} \mathrm{Head}$, Department of Psychology, KC College, Churchgate, Mumbai. \\ Corresponding author: Renu Seshadri \\ Email - renu.seshadri97@gmail.com
}

\begin{abstract}
Background: This research aims to understand the differences that may exist between male and female migrant students on variables namely Self-esteem, Life-satisfaction and Adjustment.

Methods: The sample size was 60(30 males and 30 females) aged 18-21 years, migrated to the city of Mumbai for educational purposes. Correlation(r) was calculated to understand the nature and type of relationship between Self-esteem, Life satisfaction and Adjustment.

Results: The results were found to be significant with the Self-esteem and Adjustment at 0.01 level for females and Self-esteem and Life-satisfaction at 0.01 level, Life-satisfaction and Adjustment at 0.05 level and Self-esteem and Adjustment at 0.01 level in males.

Conclusion: The results of the study could be used in everyday settings such as in schools, colleges and corporate office settings.
\end{abstract}

Keywords: Migrant, life satisfaction, self-esteem, adjustment, gender differences.

(Paper received $-6^{\text {th }}$ January 2019, Peer review completed $-27^{\text {th }}$ March 2019)

(Accepted $-28^{\text {th }}$ March 2019)

\section{INTRODUCTION}

College life is considered to be an important phase in the life of students. Not only is it a gateway to higher education but also creates an avenue for added social contact and the life building at social, health, educational and emotional levels. The expectations of liberal environment such avenues provide also brings in the expectation of fitting in and flourishing in such environment. For most college students finding friends and having a good social group becomes a part of a problem that must be resolved. The nature of this problem becomes much more complex when students migrate from other regions to enter colleges. In this situation they face a variety of challenges ranging from a different culture to a lack of familial support up to the same degree as before. Settling in a new environment gives rise to a variety of stressors which may include the fear of stigma and rejection, marginalization as well as low self-confidence and social competence [1].

As part of this exploratory study we are trying to understand and correlate the self-esteem, life-satisfaction and adjustment scores between male and female migrant students. Migration can be defined as the process of going from one country, region or place of residence to settle in another [2]. The duration of this settlement varies but for the purpose of this paper the focus is on individuals who have relocated to the metropolis of Mumbai for educational purposes.

Migration can be divided into 3 stages -

1. The decision and preparation to move 
2. Actual physical relocation

3. Post-migration: absorption of migrant within the cultural and social framework of the new environment.

For Self-esteem one of the constructs proposed by humanistic psychologist Nathaniel Branden is the disposition to experience oneself as competent to cope with challenges of life and as worthy of happiness [3].

Self-esteem forms a large part of adolescents' self-understanding and is likely to be a fluctuating and dynamic construct, susceptible to internal and external influences during adolescence. Some common factors influencing self-esteem are academic self-efficacy, relationship with peers, importance placed on physical appearance, family conflict \& gender discrimination faced, tendency for social comparison, birth order.

Life satisfaction has been defined as "a global assessment of a person's quality of life dependent on the values and criteria deemed important by the individual [4]." However, life-satisfaction is a more complex concept than the attainment of goals or the feeling good about oneself. It can be dependent on a variety of factors.

Mental health, lifestyle, physical activity, Body Mass Index (BMI), stress management skills, interpersonal connectedness are some common factors which influence life-satisfaction. According to Symonds, Adjustment can be defined as a satisfactory relation of an organism to its environment [5]. Thus, it can be said that Adjustment is a continuous process to produce a harmonious relationship between man and his environment. Adjustment to college life necessitates a range of demands differing in kind and degree and requiring lot of coping behaviours or adjustments. The students are not only concerned about academics; they are equally affected by the social and emotional changes.

Financial factors, contrast between school and college work, accommodation, academic performance, engagement in co-curricular activities are some common factors that influence adjustment. As individuals enter adolescence there are more demands in terms of changes and coping at every level including physical. We also see today that the parents as well as children are willing to explore the new possibilities of migration and for migration. It also might be helpful in an easy transition from college life to adult life.

In the course of the study the researchers have come across a number of research papers examining similar variables as this study indicating the significance of these constructs in adolescent's life. However, the research is deemed unique in the particular scope and context that it encompasses i.e. migrant students with reference to Mumbai. In addition to this, the uniqueness of the study it may also play a constructive part in understanding the overall adjustment and their corresponding accomplishments in terms of academics, interpersonal relationships, etc. in the student population: thus, helping students to focus on what they wish to accomplish in the given duration of their stay in Mumbai.

Shalini Barthwal and Mudassir Mohi-Ud-Din in their research found out that there was a significant difference among migrant and non-migrant students in overall adjustment. The male migrated students were better adjusted as compared to the female counterparts on overall adjustment especially on the health, social and emotional front [6].

Chauhan V.'s research showed that high school female students showed better overall adjustment compared to male students [7]. In Priya Sharma and Nisha Saini's study to identify the adjustment problems among college students it was revealed that Girls were average in the dimensions of health and social adjustment and unsatisfied in emotional areas while boys were average in the dimensions of social adjustment and unsatisfied in health and emotional areas. There was no significant difference between health, social and emotional adjustment of Girls and Boys. There was a significant difference between health and emotional adjustment of urban and rural college students but they did not differ significantly in social adjustment.

A longitudinal research carried out by Jan-Erik Lönnqvist to study the effects of voluntary international migration and its impact on self-esteem and life-satisfaction suggested that migration could be beneficial to some but detrimental to other types of well-being [8]. The purpose of Renshaw's study was to validate the two-continua model of mental health among college students by exploring the relation of participants' mental health status - as judged by a few indicators of psychological distress as somatization, depression, and anxiety taken in conjunction with a single indicator of psychological well-being which is life satisfaction -with three other key indicators of college student functioning namely academic achievement, interpersonal connectedness, and physical health. The findings from the frequency analysis of the mental- 
health-status variable indicated that the largest proportion of the sample was classified as mentally healthy [9].

A research study by Robert Francis Pettay (2008) explores the relationship between_a variety of health behaviours and life-satisfaction. The findings indicated_that BMI, physical activity, stress management skills and sleep were related to life-satisfaction [10]. In a study of factors shaping self-esteem among adolescent girls, it was found that poor academic self-efficacy, teasing by peers, dissatisfaction with physical appearance, family conflict and gender discrimination in the family were found linked to low self-esteem. On the other hand, satisfaction with academic performance and lesser tendency for social comparison were found to be linked with high self-esteem [11].

V. Priya and Dr. K. Muralidaran's objective was to find out the possible factors that affected self-esteem among adolescents of 17-19 age. These results pointed out that, factors such as birth order and the course selected (professional or otherwise) played a vital role.

\section{METHODOLOGY}

\section{Aim}

To understand the gender differences that may operate in migrant students on Life-satisfaction, Self-esteem and Adjustment.

\section{Hypothesis}

- There will be a correlation between self-esteem and life-satisfaction scores in males.

- There will be a correlation between life-satisfaction and adjustment scores in males.

- There will be a correlation between self-esteem and adjustment scores in males.

- There will be a correlation between self-esteem and life-satisfaction scores in females.

- There will be a correlation between life-satisfaction and adjustment scores in females.

- There will be a correlation between self-esteem and adjustment scores in females

\section{Operational Definition of Variables}

Since this was a correlational study there was no independent variable and dependent variable.

The 3 variables were -

Self-esteem: According to Rosenberg (1979) Self-esteem is the totality of an individual's thoughts and feelings with reference to himself/herself as an object [12].

Life-satisfaction: According to Neugarten, Havighurst and Tobin the term life-satisfaction refers to an individual's frame of reference and the subjective evaluations of both present and past life as an indicator of the satisfaction or happiness [13].

Adjustment: According to A.K.P. Sinha and R.P. Singh adjustment can be referred to as the local conditions and problems faced by the student population in India. Adjustment has been measured in five areas: Home, Health, Social, Educational and Emotional.

\section{Constant Variables}

- 30 Boys and 30 Girls aged 18 to 21

- English literate sample

- Residents of India

\section{Sample Size}

The sample size was 60.30 were males and 30 were females. Their ages ranged from 18 to 21 .

\section{Instruments used}

Self-Esteem - The Rosenberg self-esteem scale given by Morris Rosenberg (1965) was used to measure selfesteem levels. It is a 10 -item scale that measures global self-worth by measuring both positive and negative feelings about the self. The scale is believed to be unidimensional. All items are answered using a 4-point 
Likert scale format ranging from strongly agree to strongly disagree. The test retest reliability is 0.82 and coefficient correlation was 0.88 .

Life-Satisfaction - The Life-satisfaction scale developed by Neugarten, Havighurst and Tobin (1961) was used to measure the life-satisfaction levels among the students. It is a 13-item scale that focuses on the individual's frame of reference and the subjective evaluations of both present and past life as an indicator of the satisfaction or happiness. The reliability coefficient of correlation between the thirteen items instrument of the life satisfaction index $\mathrm{Z}$ and the earlier life satisfaction scale rating is 0.79 . Validity coefficient was 0.57 . The respondents have to indicate agreement or disagreement or "?" by placing a (x) under any of the three columns mentioned.

Adjustment - The Adjustment Inventory for college students formulated by Prof. A.K.P.Sinha and Prof. R.P.Singh was used to measure the adjustment levels. The reliability coefficient high in the overall scale and in the subscales. The inventory has 102 items that is useful to segregate normal from poorly adjusted college students in respect of five areas of adjustment namely: Home, Health, Social, Emotional and Educational.

- Home Adjustment: Low scores indicate satisfactory adjustment. Individuals scoring high tend to be unsatisfactorily adjusted toward their home surroundings.

- Health Adjustment: Low scores indicates satisfactory health adjustment and high scores unsatisfactory adjustment.

- Social Adjustment: Individuals scoring low are submissive and retiring. High scores indicate aggressive behaviour.

- Emotional Adjustment: High scores indicate unstable emotion. Individuals with low scores tend to be emotionally stable.

- Educational Adjustment: Individuals scoring high are poorly adjusted toward their curricular and co-curricular programmes. Persons with low scores are interested in the educational activities.

\section{Design}

The design of the research is quasi, repeated measures design as each participant responded to each of the questionnaire respectively. The design is quasi experimental as the sample's characteristic was that they are a migrant student.

\section{Procedure}

30 Boys and 30 Girls, aged 18 to 21 who had migrated to the city of Mumbai for educational purposes, were selected at random and with their consent were asked to fill in the questionnaires. The respondents were assured of confidentiality and privacy of results and responses and doubts if any were clarified on the spot. The respondents were also informed to request for a feedback by giving their contact details.

There was no time limit instructed, though majority of the respondents took an average of 40 minutes to complete the questionnaire. Incomplete questionnaires were not included in the data analysis.

\section{STATISTICAL ANALYSIS}

The questionnaire was manually scored and further statistical analysis was done using Microsoft Excel. Correlation (r) was calculated between two variables respectively to understand the nature and type of relationship among the variables. Various theories and research evidence were applied to understand the nature and relationship of the three variables, including the gender differences. 


\section{RESULTS}

Table 1: Summary of Descriptive Statistics based on gender (Group Data)

\begin{tabular}{|l|l|l|l|l|l|l|}
\hline & \multicolumn{2}{|l|}{ Self-esteem } & \multicolumn{2}{l|}{ Life-satisfaction } & \multicolumn{2}{l|}{ Adjustment } \\
\hline & Female & Male & Female & Male & Female & Male \\
\hline Sum & 633 & 688 & 484 & 407 & 1189 & 1452 \\
\hline Mean & 21.1 & 22.9 & 16.133 & 13.5 & 39.633 & 48.4 \\
\hline Range & 16 & 14 & 18 & 16 & 65 & 48 \\
\hline Standard Deviation & 4.146 & 3.368 & 4.717 & 4.680 & 13.593 & 13.425 \\
\hline $\mathbf{n}=$ & 30 & 30 & 30 & 30 & 30 & 30 \\
\hline
\end{tabular}

Table 2: Summary of correlation data based on gender

\begin{tabular}{|l|l|l|l|l|l|}
\hline Sr.no. & Variables & $\mathbf{n}$ & $\mathbf{d f}$ & $\mathbf{r}$ & $\mathbf{p}$ \\
\hline $\mathbf{1 .}$ & S.E-L.S (Females) & 30 & 28 & -0.12 & $\mathrm{Ns}$ \\
\hline $\mathbf{2 .}$ & L.S-Adjustment(Females) & 30 & 28 & -0.063 & $\mathrm{Ns}$ \\
\hline $\mathbf{3 .}$ & S.E-Adjustment(Females) & 30 & 28 & 0.50 & $\mathrm{p}<0.01$ \\
\hline $\mathbf{4 .}$ & S.E-L.S (Males) & 30 & 28 & -0.57 & $\mathrm{p}<0.01$ \\
\hline $\mathbf{5 .}$ & L.S-Adjustment (Males) & 30 & 28 & -0.43 & $\mathrm{p}<0.05$ \\
\hline $\mathbf{6 .}$ & S.E-Adjustment (Males) & 30 & 28 & 0.482 & $\mathrm{p}<0.01$ \\
\hline
\end{tabular}

\section{DISCUSSION}

The hypothesis states that there will be a correlation between self-esteem and life-satisfaction scores in males. The correlation(r) obtained was - 0.57 , indicating a negative moderate correlation and after referring to statistical table of correlation the data was found to be significant at 0.01 level; which means that the obtained correlation was due to true relationship and less due to chance. This could be due to:

- According to a general understanding, self-esteem and life-satisfaction are interrelated. However our results indicate that these two are possibly two separate constructs.

- From the Cognitive perspective, the typical evaluation by adolescents of their life and self-esteem may not overlap.

- The adolescent age itself is such that they are able to compartmentalize their experiences. They are full of optimism and may face every challenge believing it to be a part and parcel of that stage.

- The result could be due to an individual's personality and the way he/she lets external factors affect them.

- According to Humanistic perspective, the more the gap is between the 'real self' and 'ideal self' the more people's self-esteems are vulnerable.

The hypothesis states that there will be a correlation between life-satisfaction and adjustment scores in males. The correlation(r) obtained was - 0.43 , indicating a negative moderate correlation and after referring to statistical table of correlation the data was found to be significant at 0.05 level; which means that the obtained correlation was due to true relationship and less due to chance. This could be due to -

- The male migrant students may feel less satisfied when they have to make more adjustments in their daily lives as from a young age they are conditioned to believe that adjusting is not manly. They might gain greater satisfaction when there is relatively less adjustment required as one would understand from the social, cultural and gender aspect.

- The result also indicates that most males have been able to have a comfortable affiliation to life in Mumbai and haven't had to make substantial adjustments in their way of living.

- From a Socio-cultural perspective, it is seen that a man is not expected to engage in a lot of adjustments and consequently triumph over it. 
- In a research by Shalini Barthwal et.al on Assessment of adjustment in relation to student migration state that male migrated students were better adjusted on overall adjustment especially on the health, social and emotional front.

- The results suggest the need to assimilate the migrated students with the changed environment so that they can adjust better and work towards a high academic and other curricular achievement.

The hypothesis states that there will be a correlation between self-esteem and adjustment scores in males. The correlation(r) obtained was 0.48 , indicating a positive moderate correlation and after referring to statistical table of correlation the data was found to be significant at 0.01 level; which means that the obtained correlation was due to true relationship and less due to chance. This could be due to:

- The higher the self-esteem is, the more easy migrant males find it to adjust in a new social setting. Their strong sense of self helps them to assimilate and integrate in a new culture without feeling belittled or lost.

- A healthy idea of self-worth means that these individuals accept themselves as they are including what life has to offer them and thus feel lower social anxiety and have a good sense of selfcompetence.

- Self-esteem can also include aspects of self-efficacy which states the belief that we are capable of accomplishing the needful and acceptance of self unconditionally.

- Since this is a correlation, the result does not indicate a causal relationship.

- There is a 'spill-over effect 'i.e. higher the self-esteem higher is the adjustment.

The hypothesis states that there will be a correlation between self-esteem and life-satisfaction scores in females. The obtained correlation was -0.12 and the data was found to be not significant, indicating that the obtained correlation was more due to chance and less due to true correlation. This could be due to:

- According to the Behaviouristic perspective, 'Reinforcement is defined as any event that strengthens the behaviour it follows.'

- In this context when a person evaluates themselves positively, that itself may be rewarding or reinforcing their self-esteem which may have no spill-over effect on life-satisfaction.

- Also since we all live in a collectivistic culture life-satisfaction and self-esteem may be two independent concepts which may have no impact on each other.

- The beliefs that operate in making up of self-esteem and life-satisfaction may not match.

- The participants may have engaged in socially desirable responses. The participant coming to a new city may prioritise adjusting to the new surroundings rather focusing on their self-esteem and lifesatisfaction.

- Since the variables are measured through correlation, the nature and relationship of the variables is indicated and no causal relationship.

The hypothesis states that there will be a correlation between life-satisfaction and adjustment scores in females. The obtained correlation was -0.063 and the date was found to be not significant, indicating that the obtained correlation was more due to chance and less due to true correlation. This could be due to:

- For migrant females, life-satisfaction and adjustment are independent of each other. This phenomenon can be explained through a variety of reasons such as rationalizations that prioritise immediate adjustment over long term life-satisfaction.

- From a Socio-cultural perspective, it is expected and accepted for a woman to "adjust" with the demands of life and it is nothing unusual for them and it may have a very little spill-over effect on self-esteem.

- Since we are a collectivist culture, women are expected to be more adjusting than otherwise which may influence her self-esteem. This may explain the low correlation.

- As the individual is able to compartmentalize maladjustment in one area, it is less likely to colour the satisfaction of one's life.

The hypothesis states that there will be a correlation between self-esteem and adjustment scores in females. The correlation(r) obtained was 0.5 , indicating a positive moderate correlation and after referring to 
statistical table of correlation the data was found to be significant at 0.01 level; which means that the obtained correlation was due to true relationship and less due to chance. This could be due to:

- The result shows that females consider self-esteem and adjustment as interrelated.

- A research conducted on self-esteem by adolescents (Priya.V et.al) stated birth order and the course selected as factors influencing self-esteem can also influence adjustment.

- Also, a research on adjustment state that female students show better overall adjustment compared to male students.

\section{CONCLUSION}

Thus, from the present study it can be concluded that there was a significant difference in the levels of trust and commitment, although an insignificant difference was observed in the levels of relationship satisfaction among couples in long distance and proximally close relationship. And it was observed that, young adults in long distance relationship elicited higher levels of commitment with a mean of 115.15 as compared to young adults involved in proximally close relationship with a mean of 98.95; while lower levels of trust was observed among young adults in long distance relationship with a mean of 30.45 as compared to young adults in proximally close relationship with a mean of 38.35. But, in order for the results to be generalized to the larger population we need to take into consideration a larger sample size. Also, it would be interesting to study the gender differences pertaining to the impact of physical proximity on the dimensions of trust, commitment and relationship satisfaction.

\section{REFERENCES}

1. Chen X, Wang P, Wegner R, Gong J, Fang X, Kaljee L. Measuring social capital investment: Scale development and examination of links to social capital and perceived stress. Soc Indicators Res 2015;120(3):669-87.

2. Chesnais JC. The demographic transition: Stages, patterns, and economic implications. OUP Catalogue. 1992.

3. Mruk CJ. Self-esteem and positive psychology: Research, theory, and practice. Springer Publishing Company; 2013.

4. Huebner ES. Correlates of life satisfaction in children. School Psychol Quart 1991;6(2):103-9.

5. Pasha HS, Munaf S. Relationship of self-esteem and adjustment in traditional university students. Proc Soc Behav Sci 2013;84:999-1004.

6. Barthwal S, Mohi-Ud-Din M. A Comparative Study of Stress in Relation to Student Migration. Int J Psychol Psychiatry 2016;4(2):76-81.

7. Chauhan V. A study on adjustment of higher secondary school students of durg district. OSR J Res Method Educn 2013;2:2320-8.

8. Lönnqvist JE, Leikas S, Mähönen TA, Jasinskaja- Lahti I. The mixed blessings of migration: Life satisfaction and self- esteem over the course of migration. Eur J Soc Psychol 2015;45(4):496-514.

9. Renshaw TL, Cohen AS. Life satisfaction as a distinguishing indicator of college student functioning: Further validation of the two-continua model of mental health. Soc Indicators Res 2014;117(1):319-34.

10. Haidt J. The happiness hypothesis: Finding modern truth in ancient wisdom. Basic Books; 2006.

11. Dhillon M, Dhawan P. "But I am fat": The experiences of weight dissatisfaction in Indian adolescent girls and young women. In Women's Studies International Forum 2011 Nov 1 (Vol. 34, No. 6, pp. 539-549). Pergamon.

12. Rosenberg M, Schooler C, Schoenbach C, Rosenberg F. Global self-esteem and specific self-esteem: Different concepts, different outcomes. Amer Sociol Rev 1995;2:141-56.

13. Havighurst RJ, Neugarten BL, Tobin SS. Disengagement, personality, and life satisfaction. The Meanings of Age: Selected Papers; University of Chicago Press: Chicago, IL, USA. 1996.

$* * * * * * * * * * * * * * * * * * * * * * * * * * * * * * * * * * * *$

$$
\begin{aligned}
& \text { Acknowledgements - Nil } \\
& \text { Conflict of Interest - Nil } \\
& \text { Funding - Nil }
\end{aligned}
$$

